\title{
Neutron Multiplicity Correlations with Fission Fragment Mass and Energy from ${ }^{239} \mathrm{Pu}(\mathbf{n}, \mathbf{f})$
}

\author{
Alf Göök ${ }^{1, *, * *}$, Franz-Josef Hambsch ${ }^{1, * * *}$, and Stephan Oberstedt ${ }^{1}$ \\ ${ }^{1}$ European Commission, Joint Research Centre, Retieseweg 111, B-2440, Geel, Belgium
}

\begin{abstract}
There exists experimental evidence for strong fluctuations of the average neutron multiplicity from resonance to resonance in ${ }^{239} \mathrm{Pu}(\mathrm{n}, \mathrm{f})$. These fluctuations have been shown to impact nuclear reactor benchmarks by reducing the criticality. The fluctuating neutron multiplicity can be explained as a consequence of the competition between direct fission and the $(\mathrm{n}, \gamma \mathrm{f})$ process. However, there is also evidence for fluctuations of the fission fragment mass yields from resonance to resonance. The mass yield fluctuations may also contribute to fluctuations of the neutron multiplicity averaged over all fission fragment masses. In order to model the contribution to the neutron multiplicity fluctuations by the fission fragment mass yield fluctuations new data on the correlations between fission fragment properties and neutron multiplicities are in need. We present experiments carried out to determine prompt neutron multiplicity correlations with fission fragment masses and total kinetic energies in the reaction ${ }^{239} \mathrm{Pu}(\mathrm{n}, \mathrm{f})$. The experiment has been performed at the GELINA facility at JRC-Geel. A twin position-sensitive Frisch-grid ionization chamber is used for fission fragment identification via the double kinetic energy technique. An array of scintillation detectors is employed for neutron counting. Correlations between average neutron multiplicities and fission fragment properties have been measured with improved resolution in both mass and TKE, compared to data from the literature.
\end{abstract}

\section{Introduction}

Fluctuations of the average prompt neutron multiplicity $\left(v_{p}\right)$ from the reaction ${ }^{239} \mathrm{Pu}(\mathrm{n}, \mathrm{f})$ in the incident neutron energy range of the resolved resonances have been observed experimentally [1-3]. As seen in Fig. 1, experimental and evaluated data show $v_{p}$ fluctuations in the incident neutron energy range below $120 \mathrm{eV}$. Changes in $v_{p}$ between $1-10 \%$ at the resonance energies are evident. The fluctuations have been shown to impact nuclear reactor benchmarks by reducing the criticality [4]. A new evaluation of the prompt fission neutron spectrum (PFNS) in the thermal energy range has determined a lower value of the average neutron energy than that reported in the existing evaluated nuclear data libraries [5]. The lower average neutron energy is in agreement with independent evaluations [6]. A number of thermal-solution benchmarks have shown that the use of a softer prompt fission neutron spectrum at thermal energy, combined with new thermal neutron constants (adapted to fit with the IAEA standards), yields k-eff values that are larger than measurements by a margin that increases as the above-thermal-leakage fraction increases [7]. Therefore a reduced criticality is needed for high-leakages solutions. Accordingly, the OECD-NEA high priority request list is asking for new measurements of $v_{p}$ in ${ }^{239} \mathrm{Pu}(\mathrm{n}, \mathrm{f})$, in the incident neutron energy range from thermal to $5 \mathrm{eV}$ [8]. Fluctuations of $v_{p}$ in the region

\footnotetext{
*e-mail: gook@kth.se

** present address: KTH Royal Institute of Technology, Roslagstullsbacken 21, SE-106 91 Stockholm, Sweden

*** retired
}

of the resonances is suspected to be due to competition between direct fission and the $(\mathrm{n}, \gamma \mathrm{f})$ process, in which fission proceeds after the emission of a gamma-ray. As a consequence of the gamma-rays carrying away energy, the neutron multiplicity is lower the higher the importance of the $(\mathrm{n}, \gamma \mathrm{f})$ process is. For accurate evaluation of $v_{p}$ it is, however, also necessary to account for fluctuations of the fission fragment mass yields. Here we report on an experiment performed at the GELINA time of flight facility, where both the fission fragment mass yields as a function of the incident neutron energy and the dependence of $v_{p}$ on the fission fragment mass has been studied. We are reporting here on the results of the correlation of the average number of emitted neutrons with the fission fragment mass and total kinetic energy (TKE) release. This data provide crucial input required to model the influence of mass yield fluctuations on $v_{p}$ as a function of the incident neutron energy.

\section{Experiment}

The experiment has been performed at the GELINA neutron time-of-flight facility at the JRC Geel site. It consists of two parts: an array of proton recoil scintillators (SCINTIA) and a twin position-sensitive ionization chamber (tPIC) for fission fragments. More details about the detector setup can be found in Ref. [9]. The data acquisition is based on wave-form digitizers. A pulse from the common cathode of the tPIC triggers the data acquisition to store digital wave forms from all ionization chamber 


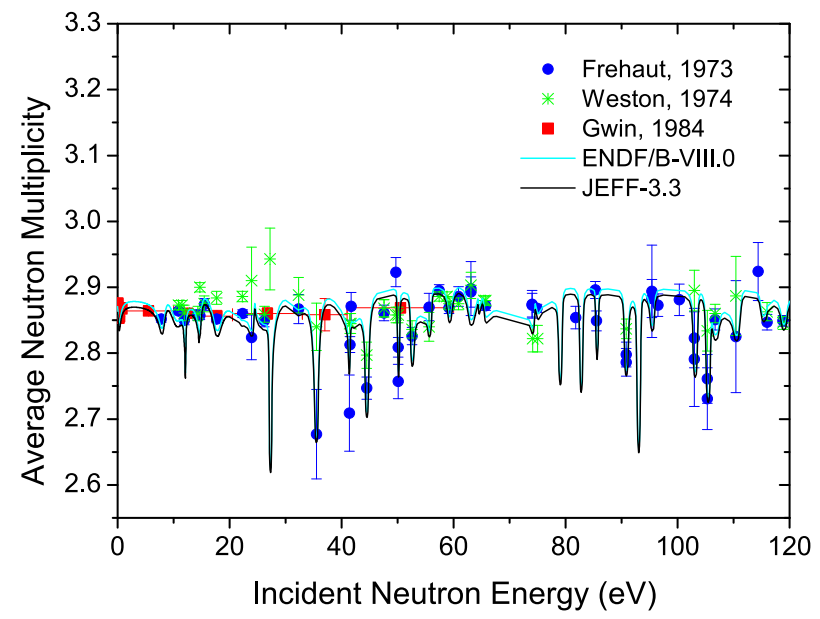

Figure 1. Fluctuations in the average neutron multiplicity as a function of the incident neutron energy in ${ }^{239} \mathrm{Pu}(\mathrm{n}, \mathrm{f})$. Experimental data from Refs. [1-3].

electrodes and scintillation detectors on disk for offline analysis. The incident neutron energy is determined via the time-of-flight technique. The main experiment was performed with GELINA operating at $800 \mathrm{~Hz}$ repetition frequency. With the incident neutron flight-path length of $8.81 \mathrm{~m}$, the lowest neutron energy accessible in this measurement is $0.26 \mathrm{eV}$. To avoid overlap of low energy neutrons from a former electron beam pulse, a $\mathrm{Cd}$ filter with an areal density of $0.7 \mathrm{~g} / \mathrm{cm}^{2}$ was placed in the beam.

\subsection{Fission Fragment Detection}

The tPIC is used for determination of fission fragment masses and energies. In principle, the detector is a twin Frisch grid ionization chamber. However, the standard anode plates are replaced by position sensing readout electrodes. The detector was developed at JRC-Geel and is described in detail elsewhere [10]. The fission target consists of a thin layer $\left(29.95 \mu \mathrm{g} / \mathrm{cm}^{2} \mathrm{Pu}\right)$ of $\mathrm{PuF}_{4}$ on a backing of gold covered polyimide. The fission target is placed in a hole in the common central cathode. The very thin target and backing allows for both of the fission fragments from a binary event to escape and ionize the gas on either side of the cathode plate. Fission fragment energies and masses are determined via the double-kinetic-energy (2E) technique. For the purpose of calibration, we have used data collected with GELINA operating at $50 \mathrm{~Hz}$ repetition frequency. This allows the selection of thermal neutron induced fission in the time of flight spectrum, which has well known characteristics. During the $50 \mathrm{~Hz}$ run the $\mathrm{Cd}$-filter was removed from the beam. As counting gas pure $\mathrm{CH}_{4}$ is used. The choice of this counting gas is motivated by its high drift velocity compared to the P-10 gas mixture, which is more commonly used with this detector type. The high drift velocity helps reducing the effect of pile-ups present due to the high alpha activity of the ${ }^{239} \mathrm{Pu}$ target. Further pile-up correction and rejection based on digital wave-form analysis was also performed.
As stated above, fission fragment masses and energies are determined via the $2 \mathrm{E}$ technique which requires prior knowledge of the average number of neutrons emitted $\bar{v}(A)$. As initial assumption we have used the evaluated data on $\bar{v}(A)$ from Wahl [11] and the parametrization

$$
\bar{v}(A, T K E)=\bar{v}(A)\left(1+\frac{\overline{T K E}(A)-T K E}{\left[\bar{v}(A)+\bar{v}\left(A_{c n}-A\right)\right] E_{\text {sep }}}\right)
$$

where $E_{\text {sep }}=8.6 \mathrm{MeV} / \mathrm{n}$ is the average energy necessary to emit a neutron [12]. The analysis was later repeated using the results for $\bar{v}(A)$ and $E_{\text {sep }}=9.02 \mathrm{MeV} / \mathrm{n}$ derived from the data. No significant changes in the results were observed between the two analyses; hence no further iteration was made. For the case when a neutron coincidence is required, an additional correction to the fragment energy according to Gavron [13] is applied. The pulse height defect of the counting gas is corrected for as described in Ref. [14], with parameters adjusted to reproduce known values of the average light and heavy fragment masses [15] and TKE [16] from ${ }^{239} \mathrm{Pu}\left(\mathrm{n}_{\mathrm{th}}, \mathrm{f}\right)$. The over-all massresolution of the present experiment is $4 \mathrm{u}$ (FWHM), it was determined by comparing the obtained mass distribution in thermal neutron induced fission with high resolution data from Geltenbort et al. [15].

\subsection{Prompt Neutron Detection}

For the purpose of studying correlations between neutrons and fission fragments it is necessary to know the neutron detection efficiency as a function of the energy of the prompt neutrons. In order to determine this, a dedicated measurement with a ${ }^{252} \mathrm{Cf}$ source placed inside the ionization chamber was performed. The prompt fission neutron spectrum (PFNS) emitted in the spontaneous fission decay of ${ }^{252} \mathrm{Cf}$ is known with an accuracy of about $1-3 \%$ in the energy range 0.15 to $11 \mathrm{MeV}$, and is considered as a neutron standard. Hence, by forming the ratio of observed and evaluated PFNS (ENDF/B.VII-1) for this decay the neutron detection efficiency can be determined. The ${ }^{252} \mathrm{Cf}$ source was deposited on a thin $\left(220 \mu \mathrm{g} / \mathrm{cm}^{2}\right) \mathrm{Ni}$ foil, and had an activity of about 3300 fissions/s. The ${ }^{252} \mathrm{Cf}$ measurement was performed under the same experimental conditions as the ${ }^{239} \mathrm{Pu}(\mathrm{n}, \mathrm{f})$ measurement, except for the incident neutron beam, which was not present. When determining the PFNS no selection of fission fragment emission angle is made. In fact, for this purpose only the signal from the tPIC's central cathode is used as a fission trigger, with a threshold adjusted to discriminate against $\alpha$-decay. The very thin targets used for the measurements and the large solid angle of acceptance of the fission fragment detector ensures that the PFNS is unperturbed by this selection.

\section{Results and Discussion}

In Fig. 2 the average neutron multiplicity per fission as a function of TKE is compared to data from Tsuchiya et al. [17]. As expected from energy balance considerations, a 


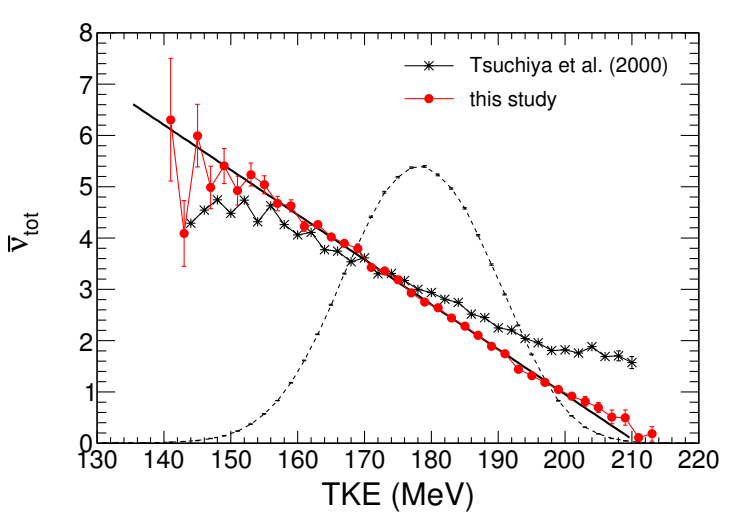

Figure 2. Average prompt neutron multiplicity per fission as a function of the fragment TKE. Data obtained in this study is compared to data from Tsuchiya et al. [17]. The dotted black line shows the shape of the fission fragment TKE distribution (without absolute scale). The full black line represent a least square fit of a straight line to the data from this study with an inverse slope $-\partial v / \partial T K E-=11.4 \mathrm{MeV} / \mathrm{n}$.

close to linear decrease of $\bar{v}$ with increasing TKE is observed. A weighted least-square fit results in an inverse slope $-\partial v / \partial T K E=11.4 \mathrm{MeV} / \mathrm{n}$. This value is close to the value observed by us in the spontaneous fission of ${ }^{252} \mathrm{Cf}$ [18], as well as in ${ }^{235} U(n, f)$ [9]. It is clear, from a visual inspection of Fig. 2 that the present data are in disagreement with the data of Tsuchiya et al. [17]. We have observed similar discrepancies with earlier experiments for the reaction ${ }^{235} \mathrm{U}(\mathrm{n}, \mathrm{f})$ [9]. As described in Ref. [9] those discrepancies are explained by better resolution in TKE from the twin ionization chamber. The same arguments presented for ${ }^{235} \mathrm{U}(\mathrm{n}, \mathrm{f})$ apply also to the present experiment. In

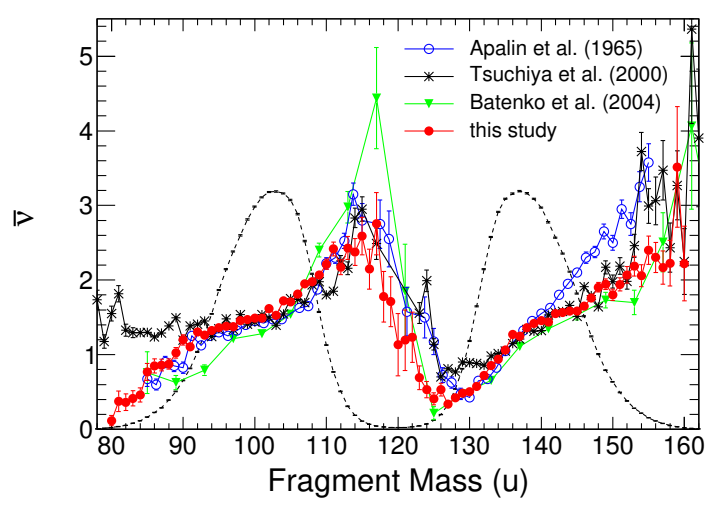

Figure 3. Average prompt neutron multiplicity per fragment as a function of the fission fragment mass. Data obtained in this study is compared to data from literature $[17,19,20]$. The dotted black line shows the shape of the fission fragment mass distribution (without absolute scale).

Fig. 3 and Fig. 4 data from the present experiment on the dependence of prompt neutron multiplicity on fission fragment mass is shown and compared to data from literature.

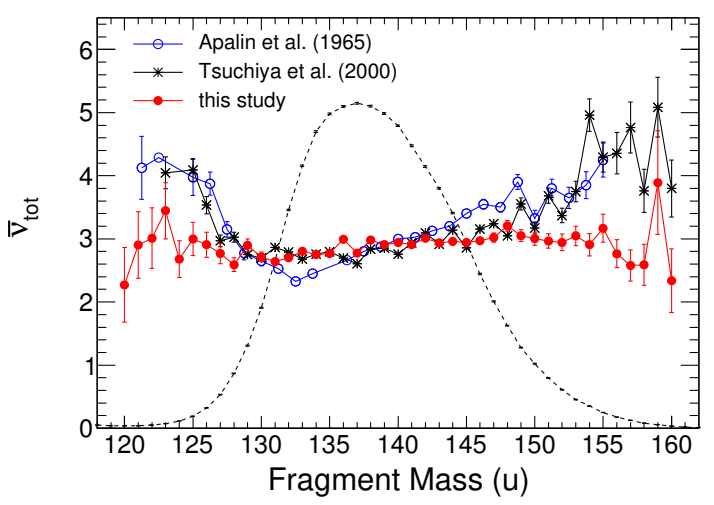

Figure 4. Average prompt neutron multiplicity per fission as a function of the heavy fission fragment mass. Data obtained in this study is compared to data from literature $[17,19]$. The dotted black line shows the shape of the fission fragment mass distribution (without absolute scale).

The neutron multiplicity per fission fragment is shown in Fig. 3, the present results show good agreement with data from Tsuchiya et al. [17] and Batenko et al. [20], in the region around the asymmetric mass peaks. In the region of symmetric mass split the present data shows a lower neutron multiplicity than the earlier experiments. The data of Tsuchiya et al. [17] shows less pronounced minima in the sawtooth like curve (around fragment masses of $80 \mathrm{u}$ and $130 \mathrm{u}$ ). This could be related to the approximately $50 \%$ poorer mass resolution in their experiment $(6 \mathrm{u})$.

Pronounced [21] and slightly weaker [22] fluctuations of the fission fragment mass yields have been found in resonance neutron induced fission on ${ }^{235} \mathrm{U}$ and ${ }^{239} \mathrm{Pu}$, respectively. This would influence $v_{p}$, if the dependence on fission fragment mass split is also strong [21]. This dependence, obtained by summing the multiplicity of light and heavy fragment, is shown in Fig. 4. In contrast to earlier experimental results $[17,19]$ the present study show a much flatter behaviour as a function of fragment mass. The difference with respect to the data of Apalin et al. (1965) can be understood in terms of a missing correction in the data analysis. Namely, the effect of the recoil of the emitted neutrons on the fission fragment mass determination. This effect was first discussed by Gavron [13], several years after the experimental data of Apalin et al. [19] was published. Hence, we may assume that Apalin et al. have not accounted for this effect. The recoil effect shows up in experimental data as an increase in neutron multiplicity for the heaviest fragments in the mass yield distribution, as well as around the symmetric mass split [13]. Consequently, the dependence of the total number of neutrons per fission will show an increased slope as a function of the heavy fragment mass as well as a large increase around symmetric mass splits.

The total number of prompt neutrons per fission does not show a strong dependence on fission fragment mass. Therefore, we may rule out fission fragment mass yield fluctuation as a major contribution to the $v_{p}$ fluctuations 
observed in resonance neutron induced fission on ${ }^{239} \mathrm{Pu}$. The more prominent contribution should be due to the competition between the direct $(n, f)$ and the $(n, \gamma f)$ reactions. Minor contribution of the fragment mass yield fluctuations can be estimated using the data presented in Fig. 4 and data on the fragment mass yields as a function of the incident neutron energy.

\section{Conclusion and Outlook}

We have reported on measurements of the average neutron multiplicity correlations with fission fragment mass and TKE in ${ }^{239} \mathrm{Pu}(\mathrm{n}, \mathrm{f})$. The correlations have been measured with improved resolution in both mass and TKE, compared to data from the literature. The new data provide crucial input required to model the influence of mass yield fluctuations on $v_{p}$ as a function of the incident neutron energy.

In order to reach the target statistical accuracy on $v_{p}$, below $1 \%$ at the resonances [8], the present experimental setup has been modified. Based on the presented results, we may rule out fission fragment mass yield fluctuations as a major contribution to the fluctuations of $v_{p}$ as a function of incident neutron energy. Therefore, the updated experimental setup will focus on measurement of the neutron multiplicity as a function of incident neutron energy alone. That is, the experiment will not attempt to measure the correlation between neutron multiplicity and fission fragment masses. We have, therefore, constructed a parallel plate fission chamber with 12 layers of ${ }^{239} \mathrm{Pu}$. Each target has an areal density of around $120 \mu \mathrm{g} / \mathrm{cm}^{2}$. This leads to an increase in the expected fission rate by a factor of 48, compared to the experiment presented here. Recently, Lynn et al. [23] published a list of resonance which are the most likely candidates to exhibit an observable (n, $\gamma \mathrm{f})$ effect, based on theoretical calculations. For these resonances the expected statistical uncertainty after half a year of measurement at GELINA has been estimated. For most of the candidate resonances the expected statistical uncertainty is well below the expected fluctuation of $v_{p}$, which lies between $5-10 \%$. The candidates for observing the $(\mathrm{n}, \gamma \mathrm{f})$ effect all have small fission cross sections. In the incident neutron energy region below $5 \mathrm{eV}$ the fission cross section is much larger, hence the expected statistical accuracy is well below $1 \%$ in this region.

\section{References}

[1] D. Shackleton, J. Trochon, J. Frehaut, M.L. Bars, Physics Letters B 42, 344 (1972)

[2] L.W. Weston, J.H. Todd, Phys. Rev. C 10, 1402 (1974)
[3] R. Gwin, R.R. Spencer, R.W. Ingle, Nucl. Sci. Eng. 87, 381 (1984)

[4] E. Fort, J. Fréhaut, H. Tellier, P. Long, Nuclear Science and Engineering 99, 375 (1988)

[5] M.T. Pigni, R. Capote, A. Trkov, V.G. Pronyaev, EPJ Web Conf. 146, 02011 (2017)

[6] R. Capote, Y.J. Chen, F.-J. Hambsch, N. Kornilov, J. Lestone, O. Litaize, B. Morillon, D. Neudecker, S. Oberstedt, T. Ohsawa et al., Nucl. Data Sheets 131, 1 (2016)

[7] C. De Saint Jean, R. McKnight, NEA/WPEC, Report NEA/NSC/WPEC/DOC(2014) 34 (2014)

[8] R. Capote, NEA nuclear data high priority request list - 99h (2018), https: //www. oecd-nea.org/ dbdata/hprl/hprlview . pl?ID=520

[9] A. Göök, F.-J. Hambsch, S. Oberstedt, M. Vidali, Phys. Rev. C 98, 044615 (2018)

[10] A. Göök, W. Geerts, F.-J. Hambsch, S. Oberstedt, M. Vidali, S. Zeynalov, Nucl. Instr. and Meth. A 830, 366 (2016)

[11] C. Wahl, Atomic Data and Nuclear Data Tables 39, 56 (1988)

[12] H. Nifenecker, C. Signarbieux, R. Babinet, J. Poitou, 3rd IAEA Symp. on the Physics and Chemistry of Fission 2, 117 (1973)

[13] A. Gavron, Nucl. Inst. and Meth. 115, 99 (1974)

[14] F.-J. Hambsch, J. Van Aarle, R. Vogt, Nucl. Instr. and Meth. A 361, 257 (1995)

[15] P. Geltenbort, F. Gönnenwein, A. Oed, Radiation Effects 93, 57 (1986)

[16] F. Gönnenwein, in: The nuclear fission process (Ed. C. Wagemans, CRC Press, Boca Raton, Florida, 1991), chap. 8, pp. 287-473

[17] C. Tsuchiya, Y. Nakagome, H. Yamana, H. Moriyama, K. Nishio, I. Kanno, K. Shin, I. Kimura, Journal of Nuclear Science and Technology 37, 941 (2000)

[18] A. Göök, F.-J. Hambsch, M. Vidali, Phys. Rev. C 90, 064611 (2014)

[19] V. Apalin, Y.N. Gritsyuk, I. Kutikov, V. Lebedev, L. Mikaelian, Nucl. Phys. 71, 533 (1965)

[20] O.A. Batenkov, G.A. Boykov, F.-J. Hambsch, J.H. Hamilton, V.A. Jakovlev, V.A. Kalinin, A.B. Laptev, V.E. Sokolov, A.S. Vorobyev, AIP Conference Proceedings 769, 1003 (2005)

[21] F.-J. Hambsch, H.H. Knitter, C. Budtz-Jørgensen, J.P. Theobald, Nuclear Physics A 491, 56 (1989)

[22] F.-J. Hambsch, I. Ruskov, Dematté, in Proc. THEORY-1 (Publications Office of the European Union, Luxemburg, 2010), p. 110

[23] J.E. Lynn, P. Talou, O. Bouland, Phys. Rev. C 97, 064601 (2018) 